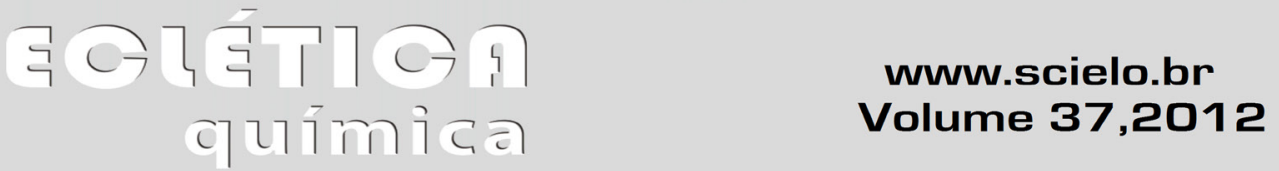

\title{
SPECTROPHOTOMETRIC DETERMINATION OF FUROSEMIDE IN PHARMACEUTICAL FORMULATIONS - A DIDACTIC APPROACH, FROM PRACTICE TO THEORY
}

\author{
Adalberto Alves da Silva Neto, Wagner Felippe Pacheco, Felipe Silva Semaan*
}

Departamento de Química Analítica, Instituto de Química da Universidade Federal Fluminense, Outeiro de São João Batista, s/n, 24020-150, Niterói - RJ, Brasil.

*felipesemaan@gmail.com

Spectrofluorimetric determination of furosemide in pharmaceutical - a didactical approach, from theory to practice.

The purpose of this paper is to present a complete possible application of simple strategies to teach basic concepts of molecular spectrofluorimetry. An ordinary anti-hypertensive drug was chosen in order to demonstrate many theoretical and practical aspects of spectrofluorimetric determinations in an accurate, precise, and inexpensive analytical lab practice. Historical aspects and fundamentals, and their contextualization for students, are also presented, being some experiments described using cheap samples.

Keywords: molecular spectrofluorimetry; furosemide; education in analytical chemistry.

\section{Introdução}

A história da química se mistura com a criação da análise química. Ainda em seus primórdios - no século XVII - os alemães usavam o termo Scheidekunst como referência a química, termo esse que significa isolar o material em seus componentes [1]. Um dos primeiros instrumentos utilizados para auxiliar as análises químicas foi a balança, embora sua descoberta remote a época dos antigos egípcios [2], as primeiras balanças confiáveis passaram a ser utilizadas apenas no século XVII, quando Antoine Lavoisier reconheceu a necessidade da precisão nas pesagens para um melhor entendimento do comportamento da matéria [3]. Essa maior precisão na medição da massa de um ou mais determinados componentes extraídos de uma amostra permitiu o crescimento da análise gravimétrica. Não só isso, em análises percentuais de minerais, a diferença entre as massas teóricas esperadas e o somatório das massas encontradas possibilitou a descoberta de novos elementos químicos, alguns deles denominados como "terras", entre eles: óxidos de zircônio, urânio, telúrio e titânio.

A medida que o conhecimento em ciência e tecnologia foi se desenvolvendo, novas técnicas de análise foram sendo criadas e/ou aperfeiçoadas. Exemplos clássicos disso são as observações iniciais de Joannes M. Marci [4] em 1648 sobre o espalhamento da luz solar nas gotículas d'água produzindo o efeito ótico conhecido como arco-íris e os experimentos de Isaac Newton sobre decomposição e recomposição da luz solar por um prisma, sendo tais exemplos a base científica que levou Robert W. Bunsen e Gustav R. Kirchhoff em 1858 a criar um espectroscópio capaz de fazer a medição das linhas de emissão de alguns elementos químicos excitados em um queimador. Isso propiciou o nascimento das técnicas de absorção e emissão atômica.

Atualmente, as técnicas instrumentais de análise são responsáveis pelo fornecimento de informações vitais para praticamente todas as áreas de ciências naturais: Química, biologia, medicina, arqueologia, metalurgia, botânica... em maior ou menor escala, todos os campos de ciência precisam das ferramentas da química analítica instrumental e de uma correta interpretação dos dados fornecidos por essas ferramentas, hoje conhecidas como técnicas instrumentais de análise.

Os métodos analíticos baseados na gravimetria e volumetria - hoje conhecidas como técnicas clássicas de análise - possuem mais relevância na aplicação de 
princípios básicos de química analítica (como reações químicas) do que em aplicações rotineiras, uma vez que as técnicas instrumentais fornecem meios para uma quantificação a níveis cada vez menores de concentração, e normalmente com um tempo mais curto de análise.

Dentro desse âmbito, as instituições de ensino superior (e muitas das instituições técnicas de ensino) têm adaptado seus currículos para incluir tais técnicas.

Devido ao nível de desenvolvimento alcançado e à conseqüente complexidade adquirida, a análise instrumental divide-se de acordo com os princípios sobre o qual a técnica é fundamentada. Por exemplo, os métodos espectrofotométricos são fundamentados na interação entre a radiação eletromagnética e a matéria. Entre eles destacam-se as espectrometrias de absorção e emissão atômicas, e as espectrometrias de absorção e luminescência molecular.

\section{A Furosemida}

A furosemida, denominada ácido 4-cloro-Nfurfuril-sulfamilantranilico, é um pó cristalino, branco e inodoro, praticamente insolúvel em água e soluções ácidas diluídas, ligeiramente solúvel em solventes orgânicos como álcoois e acetona, solúvel em soluções alcalinas diluídas [5]. A fórmula estrutural da furosemida é apresentada na Figura 1:

Figura 1: Fórmula estrutural da furosemida.

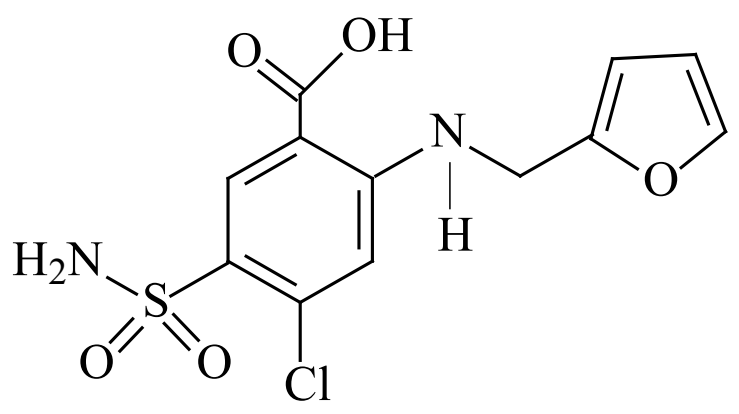

Derivada do ácido antranílico, a furosemida representa um potente diurético que, se administrado em excesso, pode gerar depleção excessiva de água e eletrólitos, devendo, portanto, ser utilizada com cuidadosa supervisão médica, havendo necessidade de ajuste de dosagem de acordo com a necessidade do paciente [6; $7 ; 8]$.

Apresentações comerciais disponíveis deste fármaco variam entre comprimidos $(20,40$ e $80 \mathrm{mg})$ e soluções injetáveis $\left(10 \mathrm{mg} \mathrm{mL}^{-1}\right)$, sendo fabricadas e vendidas por diversas indústrias farmacêuticas. Comprimidos de $40 \mathrm{mg}$ podem ser obtidos gratuitamente através do Sistema Único de Saúde (SUS).

A posologia para adultos varia entre 20 e 80 mg, em dose única pela manhã, podendo ser aumentada gradativamente, evitando-se ultrapassar $600 \mathrm{mg} \mathrm{dia}^{-1}$. Em crianças, utiliza-se dose única de $2 \mathrm{mg} \mathrm{kg}^{-1}$ de peso, podendo ser ajustada entre 1 e $2 \mathrm{mg}$, tendo-se como dosagem máxima $6 \mathrm{mg} \mathrm{kg}$.

O medicamento de referência é o Lasix ${ }^{\circledR}$ (Aventis), de ampla utilização como diurético. Com o advento dos medicamentos genéricos, aumentou-se o número de formas farmacêuticas disponíveis, exigindo maior controle da qualidade do medicamento produzido e disponibilizado ao usuário, quer seja no controle microbiológico, físico-químico ou de princípios ativos, tornando necessárias metodologias rápidas e confiáveis para aplicação nestas situações.

Por outro lado, observa-se, também a necessidade de controle rigoroso de princípios ativos, visto ser seu excesso - ou falta - gerador de danos à saúde do paciente. Dentre às reações adversas mais comuns, associadas ao uso excessivo da furosemida, pode-se citar a depleção excessiva de água e eletrólitos, como dito acima, principalmente potássio (hipocalemia), alcalose metabólica (em função da excreção de $\mathrm{H}^{+}$), hipovolemia e hipotensão, podendo ser observados efeitos mais severos como náuseas, reações alérgicas, ou ainda, surdez $[7 ; 8 ; 9]$.

Em tratamentos contínuos de crianças e adultos observa-se, ainda, a possibilidade de surgimento de tolerância e resistência ao diurético, o que pode ser superado com a administração da furosemida em associação a outros fármacos como as tiazidas. lecular.

Espectroscopia de emissão (ou luminescência) mo-

A espectroscopia de luminescência molecular é uma técnica analítica baseada no monitoramento da radiação eletromagnética (em geral luz ultravioleta, luz visível, ou infravermelho próximo) emitida por uma molécula quando essa parte de um estado excitado para o seu estado fundamental [10].

Existem diversas formas de promover a excitação da molécula para esse estado de maior energia, e é justamente essa etapa de excitação que irá diferenciar a técnica luminescente.

Caso a excitação seja por uma reação química, 
a técnica será chamada de (quimiluminescência) [11; $12 ; 13]$. No caso dessa excitação ocorrer pela passagem de uma corrente elétrica através do material, tem-se a eletroluminescência ${ }^{(14)}$. A excitação pode ser obtida pela energia estática gerada na superfície de algumas substâncias cristalinas quando essas são atritadas, resultando na emissão de luz, nesse caso, a técnica luminescente é chamada de triboluminescência [15; 16]. Outra forma de excitação é a induzida em líquidos por meio de ondas de choque (sonoluminescência) [17; 18] ou, a mais frequentemente utilizada, induzida pela incidência de luz ultravioleta, visível ou infravermelho próximo, nesse caso, a técnica é chamada de fotoluminescência [19].

Fluorescência e fosforescência são casos particulares da fotoluminescência. Ambos os processos se iniciam quando a molécula absorve um fóton de energia adequada para promover a transição de um elétron de um orbital molecular para um outro vazio, de maior energia. Normalmente o elétron sai do orbital molecular ocupado de energia mais alta (HOMO) e passa para o orbital molecular de energia mais baixa (LUMO). Como consequência dessa transição eletrônica, toda a molécula é dita como estando no estado excitado.

Quando um dos dois elétrons de spin opostos pertencentes ao mesmo orbital molecular do estado fundamental é promovido a um orbital molecular de energia mais alta, em princípio seu número de spin é mantido, de forma que o número quântico de spin $\mathrm{S}$ permanece zero $\left(S=\sum S_{i} S=\sum S_{i}\right.$, com $S_{\mathrm{i}}=+1 / 2$ ou -1/2). Como a multiplicidade "M" tanto do estado fundamental como do estado excitado é igual a $1(\mathrm{M}=2 \mathrm{~S}+1)$, ambos são chamados de estado singleto. Isso é o que ocorre na fluorescência. Nesse caso, o estado eletrônico fundamental é representado por $\mathrm{S}_{0}$, o primeiro estado eletrônico excitado por $\mathrm{S}_{1}$, e por aí vai.

$\mathrm{Na}$ fosforescência ocorre inversão de spin do estado eletrônico excitado, de forma que o número quântico de spin S muda para 1, e a multiplicidade da molécula no estado excitado muda para 3, sendo por isso chamada de estado triplete. O estado eletrônico é, então, representado pela letra $\mathrm{T}$.

A transição eletrônica singleto-tripleto é uma transição eletrônica proibida de acordo com as regras da mecânica quântica [10], no entanto, sempre há uma pequena interação entre as funções de onda de diferentes multiplicidades via acoplamento spin-orbital. Isso leva a uma pequena, porém não negligenciável, fração de transição eletrônica com inversão de spin. Na fosforescência os experimentos são realizados para aumentar essa fração.
Princípios da fluorescência molecular.

Uma vez no estado excitado, a molécula possui vários caminhos possíveis para eliminar esse excesso de energia absorvido (desativação), competindo com os fenômenos radiativos (fluorescência ou fosforescência).

Usualmente esses processos são mais bem compreendidos pelo diagrama de Perrin-Jablonski (Figura 2). Nesse diagrama, o estado eletrônico fundamental $\left(\mathrm{S}_{0}\right)$ e os excitados $\left(\mathrm{S}_{1}, \mathrm{~S}_{2}\right)$ são representados pela linha grossa, e cada um desses estados eletrônicos possui vários estados vibracionais, representados pelas linhas horizontais mais finas. As transições eletrônicas são representadas pelas setas verticais. Cada transição eletrônica é da ordem de $10^{-15} \mathrm{~s}$, tempo muito curto quando comparado à vibração das ligações na molécula (da ordem de $10^{-10}$ a $10^{-12} \mathrm{~s}$ ). Essa diferença de tempo faz com que todo o processo de absorção de luz, com consequente promoção do elétron de um orbital molecular para outro dentro da mesma molécula, ocorra sem que a molécula possua nenhuma mudança na posição relativa dos núcleos atômicos ou do ambiente molecular (princípio de Franck-Condon).

Figura 2: Diagrama de Perrin-Jablonski

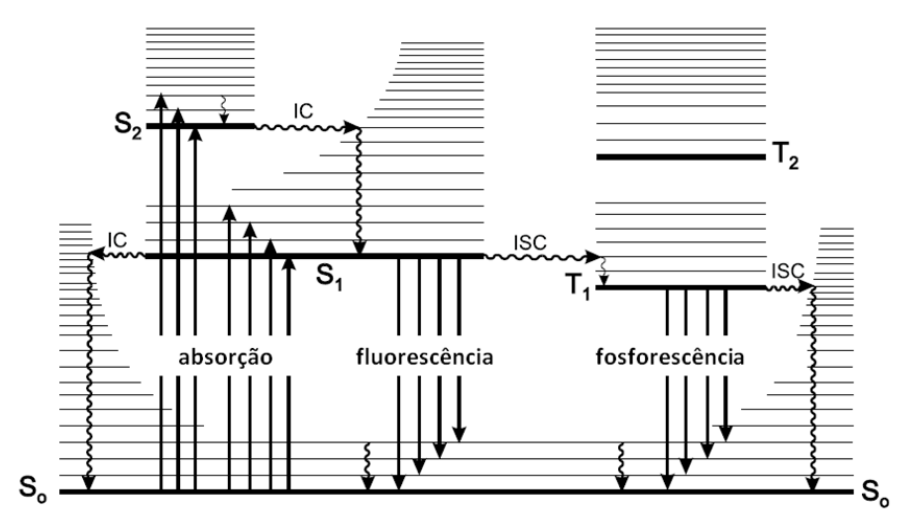

A diferença energética entre $\mathrm{S}_{0}$ e $\mathrm{S}_{1}$ é muito alta para ser atingida pela agitação térmica a temperatura ambiente, de forma que o processo de transição eletrônica normalmente se inicia do estado fundamental $\mathrm{S}_{0}$. Mesmo tendo em mente os diferentes níveis vibracionais referentes ao primeiro nível eletrônico, pode ser calculado pela equação de Boltzman que, a temperatura ambiente, a maioria absoluta das moléculas se encontra no estado vibracional de energia mais baixa, de forma que a banda de absorção é o conjunto de cada uma dessas transições a partir do estado mais baixo (tanto 
eletrônico quanto vibracional) para diferentes níveis (vibracionais e eletrônicos) de energia mais alta.

Como já foi comentado, o tempo para uma transição eletrônica é da ordem de $10^{-15} \mathrm{~s}$, e uma vez formado esse estado excitado, ele costuma se manter por cerca de $10^{-8} \mathrm{~s}$ antes de emitir luz (fluorescência). Como o estado excitado para o qual a molécula foi transferida inclui um estado vibracional mais alto, uma das possibilidades é que a molécula passe a vibrar com frequência mais alta. Essa maior frequência vibracional aumenta a possibilidade de colisões com moléculas do solvente (e também aumenta a intensidade de cada uma dessas colisões), o resultado disso é que a molécula pode transferir parte dessa quantidade de energia que ela absorveu (durante a absorção) para a molécula do solvente, se desativando, e causando um aumento imperceptível da temperatura da solução.

Esse processo se dá sem emissão de luz (e, portanto, não radiativo), e pode acontecer em níveis eletrônicos diferentes. Caso a queda de energia se dê de um estado vibracional mais alto para o fundamental, mas dentro do mesmo estado eletrônico, o processo é chamado desativação vibracional (representado pelas linhas onduladas no diagrama), caso a queda de energia se dê de um estado vibracional mais alto para o fundamental de um estado eletrônico mais baixo, o processo é chamado conversão interna (IC no diagrama).

Uma atenção especial no tempo de vida desses fenômenos deve ser dada para uma melhor compreensão das competições que estarão acontecendo. O tempo de vida do estado excitado é da ordem de $10^{-10}$ a $10^{-7} \mathrm{~s}$, da desativação vibracional $10^{-12}$ a $10^{-10} \mathrm{~s}$ e da conversão interna $10^{-11}$ a $10^{-9} \mathrm{~s}$.

Comparando o tempo de vido do estado excitado e da relaxação vibracional, observa-se que esse último processo é sempre mais rápido que a duração do estado excitado. O resultado disso, é que quando a molécula vai emitir luz, a molécula já se encontra em um nível vibracional mais baixo, e todas as emissões serão a partir do nível vibracional mais baixo do mesmo nível eletrônico.

Mais comumente ainda é que essa emissão se dê a partir do $1^{\circ}$ nível eletrônico excitado (mesmo que a molécula absorva para um $2^{\circ}$ ou $3^{\circ}$ nível eletrônico). Isso por que, como a diferença de energia entre esses níveis é muito menor que a diferença de energia entre o $1^{\circ}$ e o fundamental, o tempo para essas desativações (no caso, cruzamento interno) é menor (e, portanto a eficiência do processo é maior) do que a necessária para desativar vibracionalmente a partir do $1^{\circ}$ nível eletrôni- co.

Isso explica um ponto importante: o porquê de mesmo quando se observa em algumas moléculas vários picos de excitação (que são os análogos aos observados na espectrofotometria de absorção molecular na região do UV-Vis), só se observa um pico de emissão.

Outro ponto a ser ressaltado é que, mesmo a conversão interna sendo menos eficiente a partir do $1^{\circ}$ nível eletrônico excitado, ela ainda ocorre na maioria das moléculas. Isso explica a razão pela qual a maioria das moléculas apresenta espectros de absorção molecular, mas não apresenta espectros fotoluminescentes. Provavelmente, para pelo menos alguns desses casos, o tempo gasto na conversão interna foi menor do que o tempo do estado excitado, de modo que quando a molécula estava pronta para emitir energia na forma de luz, ela já não possuía mais esse excesso de energia (o qual foi convertido totalmente em energia térmica pela colisão com as moléculas do solvente).

Quando nenhum dos processos de desativação não radiativa acontece, a molécula irá emitir esse excesso de energia sobre a forma de luz. Caso a emissão se dê diretamente sem modificação do spin, o processo é chamado fluorescência.

Como a molécula perdeu parte da energia absorvida pela relaxação vibracional, a energia que será emitida será menor que a energia absorvida (e por tanto, o comprimento de onda será maior). $\mathrm{O}$ resultado disso é que o espectro de emissão não é sobreposto ao espectro de excitação, e sim deslocado para comprimentos de ondas maiores. A diferença entre esses picos é chamada de deslocamento de Stokes.

Em um espectro de fluorescência típico, parte do espectro de excitação e do espectro de emissão se sobrepõe, de forma que, aparentemente, uma pequena fração da solução sofre um comportamento atípico, ou seja, absorvem em comprimentos de onda maiores do que o que emite. A explicação para esse fenômeno é relativamente simples. Embora tenha representado no diagrama que toda a absorção molecular se dê a partir do estado vibracional mais baixo do estado eletrônico fundamental, uma fração muito pequena (normalmente inferior a 10 ou mesmo $1 \%$ ) se encontra em níveis vibracionais mais altos, de forma que eles podem absorver para o $1^{\circ}$ nível vibracional do $1^{\circ}$ nível eletrônico, e emitirem para um nível vibracional diferente do fundamental do nível eletrônico fundamental, e esse excesso de energia sendo perdido por relaxação vibracional. Essa pequena fração de moléculas possui um comportamento anti-Stokes. 
Outra possibilidade do composto emitir luz é sofrendo uma inversão do spin, nesse caso ocorre à fosforescência. Esse fenômeno não será abordado aqui, apenas deve ser ressaltado que ocorrendo a inversão do spin, (cruzamento intersistemas) a quantidade de moléculas disponíveis para emitir fluorescência vai diminuir, de forma que esse fenômeno irá atenuar a fluorescência.

Com base ao exposto, o presente trabalho tem por meta a apresentação de experimentos didáticos visando abordagens teórica e experimental sucintas, porém completas, do tema espectroscopia de fluorescência molecular, empregando amostras e reagentes de baixos custo e toxicidade, e fácil acesso, sem geração de resíduos que exijam tratamento especial.

\section{Procedimentos experimentais}

\section{Preparo de soluções e amostras}

Todas as soluções empregadas foram preparadas pela dissolução direta dos respectivos sólidos em água bi-destilada ou solução de hidróxido de sódio no momento do uso, não havendo, portanto, armazenamento por períodos que possivelmente comprometeriam sua aplicabilidade.

Solução de hidróxido de sódio (Merk, Alemanha) a $0,1 \mathrm{~mol} \mathrm{~L}^{-1}$ foi preparada visando a solubilização do analito (furosemida, Pharmanostra, Itália) e a extração do princípio ativo das amostras comerciais analisadas.

Tendo em vista o comportamento do analito em valores de $\mathrm{pH}$ abaixo de seu pKa [20] uma soluçãotampão de acetato de sódio (acetato de sódio p.a. e ácido acético glacial, ambos da Merk, Alemanha) com $\mathrm{pH}$ 3,3 foi preparada e empregada como meio em todas as medidas instrumentais; em casos de mais elevada acidez, como em pH 2,0 [21;22;23] o sistema se torna ligeiramente mais sensível porém, caso as medidas não ocorram imediatamente após o preparo das soluções, a hidrólise ácida pode gerar degradação significativa do analito, elevando consideravelmente as possibilidades de erros.

Solução-estoque de furosemida a $2,5 \times 10^{-3} \mathrm{~mol}$ $\mathrm{L}^{-1}$ foi preparada pela dissolução de massa adequada do analito em $\mathrm{NaOH} 0,02 \mathrm{~mol} \mathrm{~L}^{-1}$, tal estoque mostrou-se estável por até uma semana quando armazenado ao abrigo da luz e sob refrigeração.

Amostras comerciais genéricas de Furosemida ${ }^{\circledR}$ contendo nominalmente $40 \mathrm{mg}$ do princípio ativo por comprimido foram adquiridas do laboratório Teuto $\AA$; vinte comprimidos foram triturados e homogeneizados, obtendo-se como massa média de um comprimido e respectivo desvio-padrão o seguinte intervalo:

$$
\mathrm{P}_{\mathrm{m}}=(0,1994 \pm 0,0013) \mathrm{g}
$$

Tal informação foi base para a dissolução e extração da amostra visando posterior análise empregando curvas de calibração externa e adição de padrão, bem como para interpretação dos resultados obtidos.

\section{Otimização das condições experimentais}

Medidas foram realizadas empregando-se um equipamento espectrofluorímetro Cary Eclipse, da VARIAN. Partindo-se do estoque e empregando diluição direta de alíquotas adequadas do analito em tampão acetato, uma solução de furosemida a $10^{-5} \mathrm{~mol} \mathrm{~L}^{-1} \mathrm{em}$ pH 3,3 foi obtida e empregada visando otimização da obtenção de sinais analíticos; fendas de $5 \mathrm{~nm}$ (tanto para excitação quanto para emissão) foram mantidas fixas, resultando, desta forma, em espectros de excitação e emissão adequados. No caso do espectro de emissão, este foi obtido fixando-se o $\lambda_{\text {excitacão }}$ em $271 \mathrm{~nm}$, obtendo-se emissão máxima em $410 \mathrm{~nm}$, conforme apresentado na Figura 3.

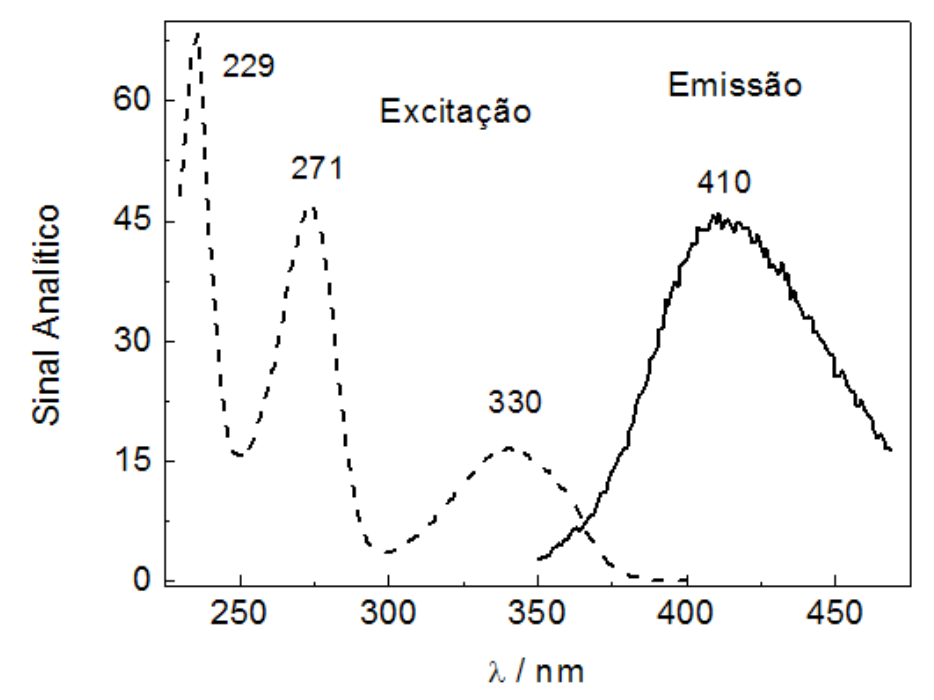

Figura 3: Espectros de excitação e emissão para solução do analito a 10-5 mol L-1 em tampão acetato pH 3,3, fendas de excitação e emissão fixas em $5 \mathrm{~nm}$.

Com base nos resultados iniciais, o sistema foi ajustado com comprimentos de onda de excitação e emissão em $271 \mathrm{~nm}$ e $410 \mathrm{~nm}$, respectivamente; fendas de $5 \mathrm{~nm}$ foram inicialmente convencionadas, sendo 
posteriormente alteradas conforme necessidade da aplicação, para melhor definição dos sinais e maior sensibilidade.

\section{Curvas analítica e adição de padrão}

Diferentes soluções foram adequadamente preparadas a partir de sistemática diluição do estoque em solução tampão acetato $\mathrm{pH} 3,3$, obtendo-se limites de detecção e quantificação de 1 e $3 \mu \mathrm{mol} \mathrm{L}{ }^{-1}$, respectivamente. Soluções de calibração entre 3 e $10 \mu \mathrm{mol} \mathrm{L}{ }^{-1}$ em furosemida (região linear) foram preparadas da mesma forma sendo imediatamente submetidas à medidas após preparo . No caso da amostra, massas adequadas do pó obtido dos comprimidos (equivalentes a meio comprimido) foram transferidas para balão volumétrico de 500,00 mL, dissolvidas e extraídas com $\mathrm{NaOH}$ $0,1 \mathrm{~mol} \mathrm{~L}^{-1}(25 \mathrm{~mL})$, o volume foi ajustado com água bi-destilada (solução A), realizou-se uma nova diluição de 5,0 mL em 100,0 mL (ajustado com tampão acetato pH 3,3; solução B). Foi feita a medida de fluorescência da solução $\mathrm{B}$, os resultados foram interpolados à curva analítica obtida. No caso dos experimentos com curvas analíticas as fendas foram alteradas para $10 \mathrm{~nm}$ tanto para excitação quanto emissão, visando melhorias em perfis de sinal e sensibilidade.

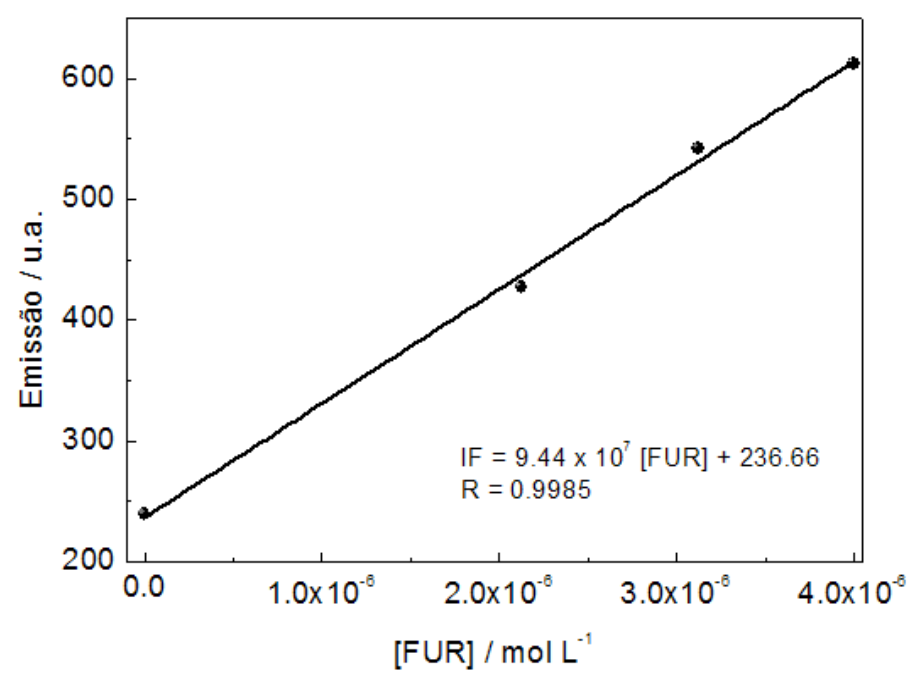

Figura 4: Curva de adição de padrão sobre amostra de Furosemida Teuto $^{\circledR}$, comprimentos de excitação e emissão respectivamente em 271 nm e 410 nm, fendas de excitação em emissão em 10 nm.

Uma segunda série de experimentos foi realizada visando avaliar a possibilidade do emprego de procedimentos de adição de padrão visando minimizar possíveis efeitos de matriz bem como reduzir o uso de reagentes e amostras. Neste caso, alíquotas constantes de 1,0 mL da amostra solubilizada (solução A) foram distribuídas em balões volumétricos, aos quais foram adicionadas alíquotas crescentes da solução-estoque padrão de furosemida, visando obter concentrações entre 2 e $4 \mu \mathrm{mol} \mathrm{L}{ }^{-1}$, tais soluções foram empregadas para a construção da curva de adição e avaliação das amostras (Figura 4). Condições de excitação e detecção foram mantidas inalteradas, embora fendas de $10 \mathrm{~nm}$ tenham sido empregadas neste caso.

\section{Resultados e discussões}

Além da abordagem da fluorescência como ferramenta analítica, alguns aspectos da técnica de espectrofluorimetria podem ser abordados com o uso da furosemida. Um deles é a regra de Kasha, no qual foi observado que o pico de fluorescência é independente do comprimento de onda de excitação, apenas a sua intensidade vai alterar. Isso pode ser observado na Figura 5, na qual vários espectros de emissão foram registrados para diferentes comprimentos de onda de excitação; valores de máximo correspondem, justamente, a cada valor de excitação.

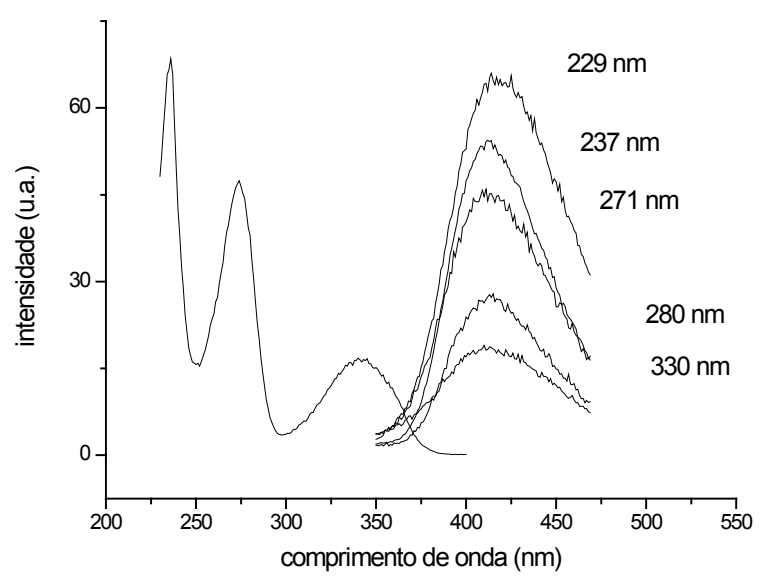

Figura 5: Aplicação da regra de Kasha para a Furosemida.

As figuras 3 e 6 apresentam espectros eletrônicos para a mesma solução de furosemida obtido pela técnica de espectrofotometria de absorção molecular na região do UV-Vis; como esperado, os espectros são análogos ao de excitação (com três picos), enquanto o espectro de emissão possui apenas um. Isso é uma observação do fenômeno de desativação do estado ex- 
citado por cruzamento intersistemas, e consequente reemissão a partir do $1^{\mathrm{o}}$ estado fundamental. Como os dois primeiros picos são de maiores energias, são eles os responsáveis para transições para níveis superiores $\left(3^{\circ}\right.$ e $2^{\circ}$, respectivamente), enquanto o $3^{\circ}$ pico de excitação é o responsável pela excitação ao $1^{\circ}$ nível eletrônico excitado, não é coincidência, portanto, que o formato do pico de emissão seja a imagem especular desse pico, o que baseado pelo princípio de Franck-Condon, representa que não houve alteração estrutural na molécula do $1^{\circ}$ nível excitado para o estado fundamental.

Também se torna interessante explorar a influência do $\mathrm{pH}$ na intensidade dos sinais analíticos obtidos. Considerando os valores já descritos de pKa's para o analito em estudo pode-se definir as distribuições de espécies em função da acidez do meio (Figura 6) de modo que, em valores de $\mathrm{pH}$ entre 0 e 2,0, é possível observar a co-existência das formas A e B, sendo a última (B) vulnerável à hidrólise ácida gerando saluamina e álcool furfurílico, conforme descrito por Cruz et al [24] e Beyers et al [25].

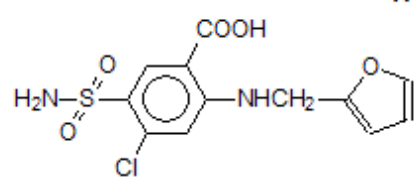

C

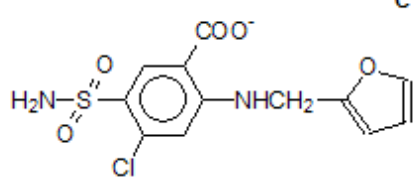

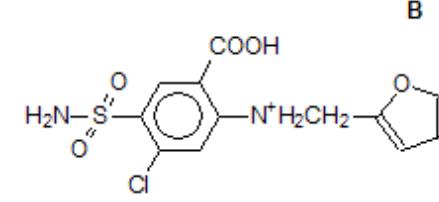

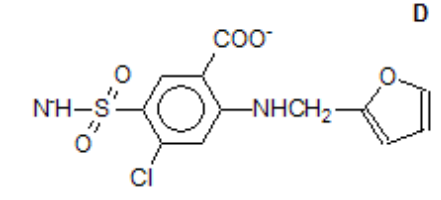

Figura 6: Possíveis formas da furosemida em função do pH.

Tal hidrólise pode ser relacionada a diferentes eventos, sendo bem descrita por Cruz et AL [24] e Beyers et al [25], um dos principais é o enfraquecimento da ligação C-N por efeitos indutivos (-I) e de deslocamento, como descrito por Beyers et al [25]. Em pH 3,3 é calculada uma distribuição de $89,91 \%$ e $10,09 \%$ para as formas $\mathrm{A}$ e $\mathrm{C}$, respectivamente; sob tais condições é possível presumir a existência de uma interação de hidrogênio intramolecular entre o grupo carboxilato e a amina secundária ocasionando uma possível contribuição de tal interação à formação e estabilização da molécula, aumentando sua rigidez e, consequentemente, sua emissão de fluorescência.

Com relação ao comprimento de onda de exci- tação é importante observar a viabilidade de emprego dos três valores observados no espectro anteriormente apresentado porém, com base às possibilidades de interferência de demais moléculas orgânicas posssivelmente presentes em amostras mais compelxas, recomenda-se o uso de $271 \mathrm{~nm}$ como comprimento de onda de excitação.

Os resultados foram obtidos em quadruplicatas, tanto por curva de calibração quanto por adição-padrão, e são apresentados na Tabela 1. Testes estatísticos $\left(\mathrm{t}_{\text {pareado }}\right)$ a um nível de confiança de $95 \%$ apontam para concordância entre os procedimentos realizados $\left(\mathrm{t}_{\text {calc. }}<\right.$ $\left.\mathrm{t}_{\text {tab. }} ; 0,58<2,78\right)$ e entre cada procedimento individual e a referência do rótulo, permitindo a escolha do mais adequado em função das necessidades.

\section{Tabela 1:}

\begin{tabular}{cccc}
\hline & Rótulo & Curva analítica* & Adição de padrão* \\
\hline Furosemida Teuto $^{\otimes}$ & $40 \mathrm{mg}$ & $(40,3 \pm 1,8) \mathrm{mg}$ & $(41,1 \pm 0,7) \mathrm{mg}$ \\
\hline${ }^{*} \mathrm{n}=4$ & & & \\
\hline
\end{tabular}

\section{Conclusões}

O uso de fármacos e medicamentos como amostras para aulas experimentais exerce grande apelo que, somado às possibilidades de exploração de conceitos tanto teóricos quanto práticos, representa uma poderosa alternativa às aulas tediosas envolvendo sondas e padrões há muito conhecidos e discutidos e sem muita aplicação na rotina profissional. No presente caso foi empregada uma molécula orgânica bioativa, a furosemida, portadora de importantes grupos funcionais e, portanto, propriedades físico-químicas e biológicas, sendo tal analito extraído da amostra comercial (comprimidos) pelo uso de simples solução de hidróxido de sódio. O custo previsto para o experimento é baixo, e a obtenção, tanto do padrão quanto das amostras, é simples. O experimento proposto tem duração relativamente curta e permite a abordagem de diferentes conceitos de forma direta e simples, sem comprometimento da qualidade da aprendizagem. Conceitos indiretamente relacionados, como estatística e normas regulatórias para produtos farmacêuticos, podem, ainda, ser explorados, tornando ainda mais eclética e realista a execução da aula nos cursos de Farmácia e Química. 


\section{Agradecimentos}

Sinceros agradecimentos à FAPERJ (Processos E-26/110.092/2010 e E-26/110.097/2010, nossos auxílios INST), à Proppi-UFF.

\section{Referência Bibliográfica}

[1] A. Yoshiko, Anal Sci. 17 (2001) 571.

[2] J. C. S. Afonso, R. M. Silva, Quim. Nova. 27 (6) (2004) 1021.

[3] N. Baccan, Química Analítica Quantitativa Elementar. Edgard Blucher LTDA, São Paulo, 3rd edn, 2001.

[4] B. Welz, M. Sperling, Atomic Absorption Spectrometry, . Wiley-VCH: Weinheim, 3rd edn, 1999.

[5] MERCK. THE MERCK Index: an encyclopedia of chemicals, drugs and biologicals. ed. Whitehouse Station, 14th edn 2001.

[6] H. L. Vlase, G. Panagopoulos, M. F. Michelis, J. Hyperntens., 16 (3) (2003), 187.

[7] H. P. Rang, M. M. Dale, J. M. Ritter, Farmacologia Guanabara Koogan. Rio de Janeiro: 6rd edn 2001.

[8] S. K. Eades, M. L. Christensen, Pediatr Nephrol. 12 (7) (1998) 603.

[9] A. R. P. Riera, Hipertensão arterial: conceitos práticos e terapêutica. Atheneu, São Paulo, 1st edn, 2000, p. 426.

[10] B. Valeur, Molecular Fluorescence: Principles and Applications.: Wiley-VCH Verlag $\mathrm{GmbH}$, Weinheim, 2001.

[11] M. Liu, Z. Lin, J. M. Lin, Anal. Chim. Acta 670 (1-2) (2010) 1.

[12] J. L. Adcock, P. S. Francis, N. W. Barnet, Anal. Chim. Acta 601 (1) (2007) 36.

[13] K. Mervartov, M. Polasek , J. M. Calatayud, J. Pharm. Biomed. anal. 45 (3) (2007) 367.

[14] H. P. M. Oliveira, R. F. Cossiello, D. Z. Atvars, L. Akcelrud, Quím. Nova. 29 (2) 2006, 26, 277.

[15] N.P. Bergeron, W.A. Hollerman, S.M. Goedeke, R.J. Moore, Int. J. Impact Eng. 35 (12) (2008) 1587.

[16] X. L. Li, Y. Zheng, J. L. Zuo, Y. Song, X. Z. You, Polyhedron. 26 (18) (2007) 5257.

[17] David J. Flannigan, Stephen D. Hopkins, Kenneth S. Suslick. J Organomet Chem. 690 (15) 20053513.

[18] M. A. Margulis, I. M. Margulis, Ultrason Sonochem. 9 (2002) 1.

[19] E. L. Wehry, Handbook of Instrumental Techniques for Analytical Chemistry, Prentice Hall PTR, 1997.

[20] F. S. Semaan, E. M. Pinto, E. T. G. Cavalheiro,
C. M. A. Brett, Electroanalysis 20 (2008) 2287.

[21] D. R. El-Wasseef, D. T. El-Sherbiny, M. A. AbuEl-Enein, S. M. El-Ashry, J. Fluoresc. 19 (2009) 817.

[22] F. S. Semaan, P. A. Nogueira, E. T. G. Cavalheiro, Anal Lett. 41 (2008) 66.[23] A. Buitrago, Avances en Química 5 (2010) 15.[24] J. E. Cruz, D. D. Maness, G. J. Yakatan, Int. J. Pharm. 2 (1979) 275.[25] H. Beyers, S. F. Malan, J. G. van der Watt, M. M. de Villiers, Drug Dev. Ind. Phar. 26 (2000) 1077. 